\title{
The Thomas Jefferson National Accelerator Facility
}

The Thomas Jefferson National Accelerator Facility (Jefferson Lab) ${ }^{[1]}$ in Newport News, Virginia, USA, is one of ten national laboratories ${ }^{[2]}$ under the aegis of the Office of Science ${ }^{[3]}$ of the U.S. Department of Energy (DOE) ${ }^{[4]}$. It is managed and operated by Jefferson Science Associates, LLC ${ }^{[5]}$. The primary facility at Jefferson Lab is the Continuous Electron Beam Accelerator Facility (CEBAF) as shown in an aerial photograph in Figure . Jefferson Lab was created in 1984 as CEBAF and started operations for physics in 1995. The accelerator uses superconducting radio-frequency ${ }^{[6]}$ (srf) techniques to generate high-quality beams of electrons with high-intensity, well-controlled polarization. The technology has enabled ancillary facilities to be created. The CEBAF facility is used by an international user community of more than 1200 physicists for a program of exploration and study of nuclear, hadronic matter, the strong interaction and quantum chromodynamics. Additionally, the exceptional quality of the beams facilitates studies of the fundamental symmetries of nature, which complement those of atomic physics on the one hand and of high-energy particle physics on the other. The facility is in the midst of a project to double the energy of the facility ${ }^{[7]}$ and to enhance and expand its experimental facilities. Studies are also pursued with a Free-Electron Laser ${ }^{[8]}$ produced by an energy-recovering linear accelerator.

\section{Continuous Electron Beam Accelerator Facility (CEBAF)}

While the proposal awarded by the Department of Energy in 1984 was to implement a conventional warm radio-frequency (rf) accelerator, with design energy of $4 \mathrm{GeV}$, the adoption of srf technology and multipass beam recirculation have set the scene for an exciting nuclear physics program over the past ten years and became the cornerstone to energy upgrades presently of $6 \mathrm{GeV}$ and in construction to $12 \mathrm{GeV}$ in 2012. The CEBAF facility (Leeman et al, 2001) has met or exceeded the expectations as originally charged by U.S. Nuclear Science Advisory Committee (NSAC), providing multi-GeV energy, with high-intensity $(200 \mu \mathrm{A}), 100 \%$ duty factor continuous wave (cw), and high-resolution beam quality $\left(\varepsilon<1 \mathrm{~mm}-\mathrm{mrad}, \Delta \mathrm{p} / \mathrm{p}<2 \times 10^{-5}\right)$. The continued evolution of technologies for polarized electron sources, cryogenic and polarized targets and electron polarimeters allow for evermore challenging and precise experiments to be performed.

\section{Polarized Electron Photo-injector}

In 1994, soon after the commissioning of CEBAF with a hot-filament thermionic electron gun, a spin-polarized electron source ${ }^{[9]}$ was implemented. This source is based on spin-polarized electron photoemission from gallium-arsenide (GaAs) semiconductor material illuminated by polarized laser light as first demonstrated by (D.T. Pierce, F. Meier, and P. Zurcher, 1975). The free spin-polarized electrons are emitted within a high-voltage DC electron gun, accelerated from rest and focused to form a beam with energy of $100 \mathrm{keV}$. Synchronous photo-injection from GaAs, where electrons are produced only at the proper time for subsequent rf acceleration, was first demonstrated at CEBAF in 1996 using an rf gain-switched diode laser (Poelker, 1995). Later, a unique system of three lasers, each successively pulsing at the $3^{\text {rd }}$ subharmonic $(499 \mathrm{MHz})$ of the accelerating rf was adopted, which allows delivery of three independent $\mathrm{cw}$ electron beams to three experimental areas simultaneously. Over the past decade, steady technological advances have led to improved electron gun performance (Sinclair et al, 2005) with exceptionally low vacuum levels $\left(10^{-12}\right.$ Torr) to protect the photocathode, novel GaAs-based semiconductors capable of producing high spin-polarization (85\%) with high quantum efficiency (1\%), and high-power (Watt), high-frequency gain-switched fiber amplified lasers that enable high-current electron beams with relatively modest

doi:10.4249/scholarpedia.102110

revision \#79409 5(7):102110

Invited by: Dr. Nicolas Alamanos, CEA, IRFU; F-91191 Gif-sur-Yvette, France[

Action editor: Dr. Nicolas Alamanos, CEA, IRFU; F-91191 Gif-sur-Yvette, France[ 
gun voltages $(\sim 100 \mathrm{kV})$. Figure shows one of the inverted design GaAs based electron gun being assembled.

\section{Superconducting Radiofrequency Acceleration}

The timely maturation of srf technology in the mid 1980s, combined with the challenging limitations of using warm-rf linacs to achieve the required beam-quality existing at CEBAF today, resulted in one of the most innovative aspects of CEBAF: the choice of using srf acceleration and demonstrated implementation of srf cavities on a production scale. Following worldwide assessment, srf cavities designed and developed at Cornell University became the basis of the 338 cavities, originally five-cell now along with higher-performance seven-cell, that comprise a $60 \mathrm{MeV}$ injector and two $600 \mathrm{MeV}$ linacs. The cavities, fabricated from niobium, are assembled in thermally insulated cryostats and cooled by a cryogenic network supplied with liquid helium chilled at a central refrigeration point. Operating since 1994, Jefferson Lab's cryogenic Central Helium Liquefier ${ }^{[10]}$ is the world's largest single $2 \mathrm{~K}$ helium refrigerator. The refrigerator maintains 70,000 liters of liquid helium at a vacuum pressure of 0.031 atmospheres within superconducting cryomodules, which are used to accelerate the CEBAF accelerator electron beam. While the choice of srf technology suppressed costs associated with a multi-hundred-megawatt warm-rf system, it perhaps more significantly provided a straight-forward path for cw operation and the ability to attain the small relative energy spread $\left(\Delta \mathrm{p} / \mathrm{p}<2 \times 10^{-5}\right)$ that exists at CEBAF today. The evolution of the quality of Jefferson Lab's superconducting cavities over of the years is shown in Figure .

\section{Beam Recirculation}

CEBAF similarly represents the first large-scale implementation of beam recirculation as shown diagramtically in Figure . Here the electron beam passes multiple times through both linacs, gaining energy on each pass. A novel aspect of recirculation is that even while the electron beam energy grows, the electron velocities all remain nearly identical to the speed of light so that multiple beam energies may be simultaneously co-accelerated. The magnetic recirculation system then has a clear job to bend (dipoles) and focus (quadrupoles) the electron beam between each linac, assuring the distribution of electrons remains achromatic (no energy dependence) and isochronous (no time dependence). The implementation of nine independent recirculation arcs allows path length controls as the machine seasonally expands and contracts and also allows for independent control to address emittance effects due to synchrotron radiation of the higher-energy passes.

\section{Beam Extraction and the Switchyard}

All beams can attain the maximum possible beam energy by making five recirculations, however, on each of the first four recirculations any one of the three beams may also be extracted from the accelerator at lower energy. This is accomplished by rf deflecting cavities properly phased to extract one bunch train toward the beam switchyard while the others continue on for additional recirculation. A photo of the rf deflecting cavities is shown in Figure . Once extracted, each beam is similarly deflected into the proper location of a septum Lambertson magnet. This is a specialized magnet which further separates the three electron beams into three unique beam lines, each terminating at one of the experimental halls A, B or C. In this way, one experimental hall may uniquely receive a lower pass energy, yet all three halls may receive the maximum beam energy.

\section{Nuclear Physics Program (1998 - 2012)}

The experimental nuclear physics program at Jefferson Lab is carried out in three experimental halls: Hall A ${ }^{[11]}$, Hall $\mathrm{B}^{[12]}$, and Hall $\mathrm{C}^{[13]}$. The halls all make use of CEBAF's high-energy electrons to carry out experiments on various nuclear targets with electron energies from a few hundred $\mathrm{MeV}$ up to $6 \mathrm{GeV}$. With the use of radiators, experiments can also be done with either tagged or untagged photons. Using a Wien filter at the polarized source, the accelerator can always provide full polarization to one experimental hall. By carefully selecting the exact combination of energies and recirculation passes, nearly full polarization can be provided to all three experimental 
areas simultaneously (Grames et al. 2004).

Each of the halls has a unique complement of experimental equipment to allow it to focus on different aspects of understanding the transition from hadronic to partonic degrees of freedom. To date, over one hundred different nuclear physics experiments have been performed at Jefferson Lab (list of completed experiments ${ }^{[14]}$ ). These experiments make use of a wide variety of nuclear targets. These targets can be as simple as a thin foil of carbon or as complex as a high power liquid hydrogen target.

\section{Hall A}

Jefferson Lab's Hall A ${ }^{[15]}$ was designed to precisely study electro- and photo-induced reactions at very high luminosity and with excellent momentum and angular resolution (Alcorn et al. 2004). The central components of Hall A are two nearly identical, 6 msr High Resolution Spectrometers (HRS), which allow the vertical drift chambers ${ }^{[16]}$ in the focal plane of these spectrometers to provide a reconstructed momentum resolution of better than $1 \times 10^{-4}$ full-width at half-maximum. To compliment the tracking, a variety of Cherenkov counters, scintillators and lead-glass calorimeters can be installed to provide customized particle identification for each experiment. The facility has been operated successfully at a luminosity in excess of $10^{39}$ nucleons cm $\mathrm{cs}^{-2}$. Hall A's research program is aimed at a variety of subjects, including nucleon spin structure functions ${ }^{[17]}$, parity violation ${ }^{[18]}$, nucleon form factors ${ }^{[19]}$ and properties of the nuclear medium, such as short-range correlations ${ }^{[20]}$ and medium modifications ${ }^{[21]}$. A summary of Hall A's top cited papers can be found here ${ }^{[22]}$.

Figure shows a fish eye view of the Hall A collaboration standing in front of one of the two high resolution spectrometers. Each spectrometer can be remotely rotated from 12.5 degrees to angles up to 140 degrees. They can be operated in either positive or negative polarity with the left spectrometer able to accept momenta of up to 4.2 $\mathrm{GeV} / \mathrm{c}$ and the right up to $3.2 \mathrm{GeV} / \mathrm{c}$. The detector package can be seen rolled out from its protective hut in the upper left of the photo.

$===$ Hall $\mathrm{B}===$

Jefferson Lab's Hall B ${ }^{[23]}$ houses the CEBAF Large Acceptance Spectrometer(CLAS) (Mecking et al. 2003), which is used to study electron-production reactions and, with a radiator and tagging system, photo-production reactions. While CLAS opperates at a lower luminousity than Jefferson Lab's other experimental halls, $1 \times 10^{34}$ nucleons $\mathrm{cm}^{-2}$ $\mathrm{s}^{-1}$, it provides detection of particles over a nearly complete sphere, making it ideally suited for studying reactions with many particle final states. A six-coil toroidal magnet provides the magnetic field for deflecting charged particles whose trajectories are determined by reconstruction with drift chambers. For these particles, CLAS can provide a momentum resolution of approximately $0.5 \%$ while Cherenkov counters, time-of-flight scintillators and electromagnetic calorimeters provide particle identification. CLAS has been used to study the structure and interactions of mesons, nucleons and nuclei using polarized and unpolarized electron and photon beams and targets. These measurements include determining deep inelastic structure of the nucleon ${ }^{[24]}$, understanding the delta resonance ${ }^{[25]}$, deeply virtual Compton scattering ${ }^{[26]}$, and probing nucleon-nucleon short-range correlations ${ }^{[27]}$. One of the major programs aims at complete determination of the $\mathrm{N}^{*}$ resonance excitations of the nucleon. In addition to experiments with CLAS, Hall B has been used with special equipment to determine the lifetime of the neutral pion (Primex) ${ }^{[28]}$ and to study rare radiative decays of the phi meson (Radphi) ${ }^{[29]}$. A summary of Hall B's publications can be found here ${ }^{[30]}$.

Figure shows a photo of the CLAS system in Hall B. While Hall B is the smallest of the three experimental halls, its detector package covers a far larger solid angle then the equipment in the other two halls, giving Hall B a unique view of many particle final state reactions. 


\section{Hall C}

The Hall $\mathrm{C}^{[31]}$ end-station at Jefferson Lab houses the High Momentum Spectrometer (HMS), which is composed of four superconducting magnets as well as a Short Orbit Spectrometer (SOS). These spectrometers can detect electrons, pions, protons and kaons with momenta up to $7 \mathrm{GeV} / \mathrm{c}$ and $2 \mathrm{GeV} / \mathrm{c}$ respectively. These devices are used to further understand the structure of baryons and mesons ${ }^{[32]}$ and to study the effects of the nucleus ${ }^{[33]}$ on nucleon properties and of the propagation of particles through the nuclear medium ${ }^{[34]}$. In addition to this standard equipment, additional large spectrometers or detectors are often temporarily installed in Hall $\mathrm{C}$ to be used stand-alone or in conjunction with the HMS and SOS. Measurements conducted with the additional equipment include searches for the strange quark content of the nucleon ${ }^{[35]}$ and new physics through parity-violating electron scattering ${ }^{[36]}$, mapping of charge distributions in the nucleon via measurement of form factors and study of the structure of hypernuclei ${ }^{[37]}$. A complete summary of Hall C's publications can be found here ${ }^{[38]}$.

Figure shows a top view of Hall $\mathrm{C}$. The electron beam runs from the bottom right to the upper left, with the cylindrical scattering chamber housing the target at bottom center. On the upper right the HMS spectrometer can be seen with its blue colored superconducting dipole and yellow detector hut. On the left side, the orange dipole of the SOS can be seen along the top of it detector hut.

\section{CEBAF 12 GeV Upgrade Project}

As with nearly every accelerator, before it was in operation, physicists were planning for the next improvement; in the case of CEBAF that entailed the doubling of the energy of the machine and extensive upgrades to the existing experimental equipment and the creation of a fourth experimental hall, Hall D, that will allow experimentalists to continue to map out the transition from hadronic to partonic degrees of freedom with over thirty experiments already planned: (list of approved $12 \mathrm{GeV}$ experiments ${ }^{[39]}$ ).

\section{Doubling CEBAF Beam Energy}

While the existing srf cavities operate on average at $50 \%$ above original specification to reach $6 \mathrm{GeV}$, an advanced class of srf cavities each four times more powerful than the earlier design are planned for the accelerator upgrade. By adding 5 new cryomodules which contain a total 40 of these new high-performance 7-cell cavities to each linac and doubling the helium refrigeration capacity, the accelerator will achieve $2.2 \mathrm{GeV} /$ pass $(1.1 \mathrm{GeV} / \mathrm{linac})$. Modification to the existing recirculation magnets and beam extraction system will allow each of the existing experimental end stations to then receive an $11 \mathrm{GeV}$ beam. A new tenth arc will be added to reach the top energy of $12 \mathrm{GeV}$ to drive a new experimental photon program in the new Hall D. An overview of these improvements is shown in Figure .

\section{Hall A: Large Installation Experiments}

During Jefferson Lab's $6 \mathrm{GeV}$ period, Hall $\mathrm{C}$ was the location for large installation experiments. After the $12 \mathrm{GeV}$ upgrade, Hall A will assume this role. Thus far, two key large equipment programs for Hall A have been approved by the Jefferson Lab Program Advisory Committee. One will use a large-acceptance device know as super-BigBite ${ }^{[40]}$ to study the electromagnetic form factors at large squared four-momentum transfer $\mathrm{Q}^{2}$. The other large installation experiment would be a high precision, parity-violation, measurement of electron-electron scattering known as Moller ${ }^{[41]}$. There are also plans to use Hall A for a unique study of the structure of the mirror nuclei tritium and helium-3. 


\section{Hall B: CLAS12}

The Hall B CLAS spectrometer will be upgraded to focus on forward-angle scattering experiments with an upgraded spectrometer system called CLAS12. The new forward detectors in CLAS12 will provide improved electron-pion separation, while the new central detector region, based on a compact 5 Tesla solenoid magnet with silicon strip detectors and drift chambers, will provide the particle tracking. The upgraded Hall B facility will focus on mapping the nucleon's 3-dimensional structure through measurements of deeply exclusive and semi-inclusive processes. A summary of the Hall B $12 \mathrm{GeV}$ physics program can be found here ${ }^{[42]}$.

Figure shows a CAD image of the CLAS12 system. A high threshold Cherenkov counter (HTCC) is located in front of a superconducting toroidal magnet. This is followed by three regions of drift chambers, a low theshold Cherenkov counter (LTCC), and finally an electromagnetic calorimeter (EC). At the heart of the new CLAS12 spectrometer is a 5 Telsa solenoid with a barrel strip tracker (BST) and forward silicon tracker (BST).

\section{Hall C: SHMS}

As part of the upgrade, Hall $\mathrm{C}$ will get a second high-resolution spectrometer. This new device will replace the large-acceptance Short Orbit Spectrometer (SOS) and provide Hall C with a spectrometer capable of reaching a momentum of greater then $11 \mathrm{GeV} / \mathrm{c}$. This new spectrometer, known as the Super High Momentum Spectrometer (SHMS), along with the current HMS spectrometer, will turn Hall C into a classic two-spectrometer facility, similar to Hall A's setup during the $6 \mathrm{GeV}$ period. The planned experimental program will focus on deep inelastic form factors, short-range correlations, and nucleon and pion form factors. An important facet of its work will be the separation of longitudinal and transverse virtual photon cross sections in semi-inclusive measurements.

Figure shows how Hall C will look in the future with the new Super High Momentum Spectrometer (SHMS) shown in the upper left replacing the short-orbit spectrometer.

\section{Hall D: GlueX}

Hall D's ${ }^{[43]}$ primary focus will be on understanding the gluons that confine the quarks in a nucleus. Confinement is a unique property of quantum chromodynamics (QCD), yet it is still not well understood. To improve our knowledge of confinement, Hall D's GlueX collaboration ${ }^{[44]}$ will focus on searching for exotic hybrid mesons that explicitly require the gluonic degrees of freedom in order to exist. As photoproduction is expected to be the most effective way to produce such exotic hybrids, Hall D will be uniquely suited to find these rare particles as Jefferson Lab's electron beam will pass through a radiator producing a high-energy beam of polarized photons via coherent bremsstrahlung. The Hall D spectrometer will consist of a superconducting solenoid and hermetic detector system.

Figure shows the design of the GlueX spectrometer. A superconducting solenoid will surround the target and detectors which consist of a barrow calorimeter (BCAL), wire tracking chambers (CDC \& FDC), time-of-flight scintillators (TOF) and a lead-glass calorimeter (FCAL).

\section{Free-Electron Laser}

The superconducting technology that Jefferson Lab pioneered for nuclear physics research with electron beams permitted the accelerator physicists at TJNAF to contemplate a revolutionary device with light. Funded by the Office of Naval Research, the Jefferson Lab Free-Electron Laser (FEL) ${ }^{[45]}$ is the only cw-FEL currently (mid-2010) in operation, providing intense, powerful beams of laser light that can be tuned to a precise color or wavelength. Free-electron lasers absorb and release energy at any wavelength, because the electrons are freed from atoms. This key feature enables the FEL to be more flexible than conventional lasers. The Jefferson Lab FEL can produce extremely intense pulses (over $10 \mathrm{~kW}$ in the infrared) of light at $\mathrm{MHz}$ repetition rates. The FEL is powered by an energy-recovering linac. In this design, electrons are accelerated by a linac, sent through a magnetic wiggler in an optical cavity to produce photons, and directed back through the same linac but 180 degrees out of phase. There, 
they give up most of their energy to a new batch of electrons, making the process highly efficient.

Jefferson Laboratory is presently proposing JLAMP (JLab AMPlifier) ${ }^{[46]}$, a 4th generation light source that will be capable of producing short 50-100 fs optical pulses to study ultrafast processes in the range of 10-100 eV to support atomic, chemical and materials research. The existing JLab FEL serves, by virtue of its cw operation, as a springboard to produce average brightness many orders of magnitude larger than exists today. Such a small, fast, bright tool is intended to enable breakthrough class experiments, enabling the understanding and creation of novel materials and devices primarily aimed at improving the use of energy without harming the environment.

\section{Acknowledgments}

We would like to thank our numerous colleagues from the past and the present whose work is represented by this article. In particular we were helped in the development of the text by Dana Arenius, Volker Burket, Eugine Chudakov, Kandice Carter, Charlie Reece, Marcy Stutzman and Steve Wood.

\section{References}

Alcorn, J et al. (2004). Basic Instrumentation for Hall A at Jefferson Lab. Nuclear Instruments and Methods A522: 294-346.

Grames, J et al. (2004). Unique Electron Polarimeter Analyzing Power Comparison and Precision Spin-Based Energy Measurement. Physical Review Special Topics - Accelerators and Beams 7: 042802.

Pierce, DT; Meier, F and Zurcher, P (1975). Negative electron affinity GaAs: A new source of spin-polarized electrons Appl. Phys. Lett. 26: 44-49.

Leeman, CW; Douglas, DR and Krafft, GA (2001). The Continuous Electron Beam Accelerator Facility: CEBAF at the Jefferson Laboratory. Annual Reviews of Nuclear Particle Science 51: 412-450.

Mecking, B et al. (2003). The CEBAF Large Acceptance Spectrometer (CLAS). Nuclear Instruments and Methods A503: 513-553.

Poelker, M (1995). High Power Gain-switched Diode Laser Master Oscillator and Amplifier. Applied Physics Letters 67: 2762-2764.

Sinclair, C K et al. (2007). Development of a High Average Current Polarized Electron Source with Long Cathode Operational Lifetime. Physical Review Special Topics - Accelerators and Beams 10: 21.

\section{Further Information and Reading}

See the Jefferson Lab home page ${ }^{[47]}$.

Hernandez-Garcia, C; O'Shea, PG and Stutzman, ML (2008). Electron Sources for Accelerators. Physics Today 61N2: 44-49. ${ }^{[48]}$

Higinbotham, D; Piasetzky, E and Strikman, M (2009). Protons and Neutrons Cosy up in Nuclei and Neutron Stars. CERN Courier 49N1: 22-44. ${ }^{[49]}$

Lung, A and Rode, C (2009). Jefferson Lab Starts Its 12 GeV Physics Upgrade. CERN Courier 49N3: 15-18. [50] 


\section{Outgoing Links}

[1] http://www.jlab.org/

[2] http://www.science.doe.gov/National_Laboratories/index.htm

[3] http://www.science.doe.gov/

[4] http://www.energy.gov/

[5] http://www.jsallc.org/

[6] http://www.scholarpedia.org/article/

The_Thomas_Jefferson_National_Accelerator_Facility\#Superconducting_Radiofrequency_Acceleration

[7] http://www.scholarpedia.org/article/The_Thomas_Jefferson_National_Accelerator_Facility\#CEBAF_12_GeV_Upgrade_Project

[8] http://www.scholarpedia.org/article/The_Thomas_Jefferson_National_Accelerator_Facility\#Free-Electron_Laser

[9] http://www.jlab.org/accel/inj_group

[10] http://www.jlab.org/accel/eng/cryo/

[11] http://www.scholarpedia.org/article/The_Thomas_Jefferson_National_Accelerator_Facility\#Hall_A

[12] http://www.scholarpedia.org/article/The_Thomas_Jefferson_National_Accelerator_Facility\#Hall_B

[13] http://www.scholarpedia.org/article/The_Thomas_Jefferson_National_Accelerator_Facility\#Hall_C

[14] http://www.jlab.org/exp_prog/generated/6GeV/completed.html

[15] http://hallaweb.jlab.org

[16] http://www.slac.stanford.edu/spires/find/hep/www?j=NUIMA,A474,108

[17] http://www.slac.stanford.edu/spires/find/hep/www?eprint=nucl-ex/0509007

[18] http://www.slac.stanford.edu/spires/find/hep/www?eprint=nucl-ex/0402004

[19] http://www.scholarpedia.org/article/Nucleon_Form_factors

[20] http://hallaweb.jlab.org/experiment/E01-015/Science/index.html

[21] http://www.slac.stanford.edu/spires/find/hep/www?eprint=nucl-ex/0211022

[22] http://hallaweb.jlab.org/publications/

[23] http://www.jlab.org/Hall-B

[24] http://www.slac.stanford.edu/spires/find/hep/www?eprint=nucl-ex/0605028

[25] http://www.slac.stanford.edu/spires/find/hep/www?eprint=hep-ex/0110007

[26] http://www.slac.stanford.edu/spires/find/hep/www?eprint=hep-ex/0107043

[27] http://www.slac.stanford.edu/spires/find/hep/www?eprint=nucl-ex/0508026

[28] http://www.jlab.org/primex/

[29] http://www.jlab.org/ radphi/

[30] https://clasweb.jlab.org/membership/publist.php

[31] http://www.jlab.org/Hall-C

[32] http://www.slac.stanford.edu/spires/find/hep/www?eprint=nucl-ex/0607007

[33] http://www.slac.stanford.edu/spires/find/hep/www?eprint=arXiv:0904.4448

[34] http://www.slac.stanford.edu/spires/find/hep/www?r=JLAB-PHY-02-189

[35] http://www.slac.stanford.edu/spires/find/hep/www?eprint=nucl-ex/0506021

[36] http://www.slac.stanford.edu/spires/find/hep/www?eprint=hep-ex/0308049

[37] http://www.slac.stanford.edu/spires/find/hep/www?eprint=nucl-ex/0408011

[38] http://hallcweb.jlab.org/publications/

[39] http://www.jlab.org/exp_prog/generated/12GeV/approved.html

[40] http://hallaweb.jlab.org/12GeV/SuperBigBite/

[41] http://hallaweb.jlab.org/12GeV/Moller/

[42] http://arxiv.org/abs/0810.4718

[43] http://www.jlab.org/Hall-D

[44] http://www.gluex.org/

[45] http://www.jlab.org/FEL

[46] http://www.jlab.org/FEL/jlamp.html

[47] http://www.jlab.org

[48] http://www-spires.slac.stanford.edu/spires/find/hep/www?j=PHTOA,61N2,44

[49] http://www.cerncourier.com/cws/article/cern/37330

[50] http://cerncourier.com/cws/article/cern/38296 